\title{
The effect of reliability, content and timing of public announcements on asset trading behavior ${ }^{\text {th }}$
}

\author{
Brice Corgnet ${ }^{\mathrm{a}, \mathrm{b}}$, Praveen Kujal ${ }^{\mathrm{c}}$, David Porter ${ }^{\mathrm{d}, *}$ \\ a Business Department, Universidad de Navarra, Spain \\ ${ }^{b}$ LESSAC ESC Dijon, France \\ c Department of Economics, Universidad Carlos III de Madrid, Spain \\ d Argyros School of Business and Economics, Chapman University, United States
}

\begin{tabular}{l}
\hline JEL classification: \\
C92 \\
G12 \\
Keywords: \\
Experimental asset markets \\
Bubbles \\
Market communications \\
Bounded rationality
\end{tabular}

\section{A B S T R A C T}

Financial markets are overwhelmed by daily announcements. We use experimental asset markets to assess the impact of releasing public messages with different levels of reliability on asset prices. Subjects receive qualitative announcements in predetermined trading periods that are either preset by the experimenter, randomly selected, or determined by past asset market prices. We find that messages can play a significant role in bubble abatement, or rekindling. The preset message, "The price is too high," decreases the amplitude and duration of bubbles for inexperienced subjects. Announcements that depend on the actual level of mispricing reduce bubble magnitude. Meanwhile, a preset or random message, "The price is too low," prevents experienced subjects from abating bubbles. We account for the effect of public messages by showing that they significantly reduce inconsistent ("irrational") trading behavior.

\section{Introduction}

"When you get this far away from a recession, invariably forces build up for the next recessions, and indeed we are beginning to see that sign."

Alan Greenspan, Hong Kong business conference, February 26th, 2007.

While the reaction of stock markets to the release of quantitative data has been analyzed extensively in the experimental literature (Forsythe et al., 1982; Plott and Sunder, 1982, 1988; Sunder, 1992), the response of markets to qualitative statements such as stock analysts' recommendations or central bankers' speeches has received less attention. ${ }^{1}$ The importance of such statements is highlighted by the market reaction to the speech made by Alan Greenspan (February 2007) after he

\footnotetext{
5. The authors acknowledge financial support from Grant ECO2008-00977/ECON from the Spanish Ministry of Education. Kujal acknowledges financial support from the Instituto Universitario de Economía, Consolider-Ingenio 2010 and the Comunidad de Madrid (grant Excelecon). Seminar participants at University of Granada and JEE 2008 conference-Dijon gave helpful comments.

* Corresponding author. Tel.: +1 7149976915 .

E-mail address: dporter@chapman.edu (D. Porter).

Researchers have recently stressed the distinction between quantitative data and qualitative statements opposing hard and soft information. Hard information is quantitative, easy to store and transmit in impersonal ways whereas soft information is often communicated in text and includes opinions, ideas, rumors, economic projections, statement of management's future plans, and market commentary (Petersen, 2004).
} 
had stepped down as chairman of the Federal Reserve Bank. The speech had a domino effect resulting in the fall of all major stock markets worldwide.

A natural question to ask would be why markets react in this manner. Defenders of full rationality may argue that Greenspan's speech had an informative content as he may have had access to privileged information about the possible evolution of US interest rates. Other commentators stress that Greenspan did not have credible information on which to base his assertions. More importantly, he did not have power to take action at the Fed anymore. The following statements stress that financial analysts were concerned that Greenspan's announcement could generate effects that would destabilize financial markets.

“He's setting a very bad precedent." "I find it unusual that he's been talking so much." Andrew Brenner, market analyst at MAN Financial

"But should we say everyone has freedom of speech except former chairmen of the central bank?" Marc Chandler, senior currency strategist at Brown Brothers Harriman in New York

This episode stresses the need to understand the reaction of financial markets to messages that apparently contain little information. Using real market data is rendered impossible by the multiplicity and simultaneity of messages reaching the market. The message space can, however, be controlled in an experimental setting. We can choose messages with some specific characteristics, something that would be impossible to control in the real world. For example, we can decide when to release a message (affecting its timing), what to put in it (affecting its content), or vary its source (affecting its reliability). We define the reliability of a message as the likelihood that the announcement is actually true. ${ }^{2}$

In this paper we analyze the effect of messages on asset prices in a controlled experimental asset market. We release messages in standard experimental asset markets first described in Smith et al. (1988), hereafter SSW. We find support for the hypothesis that apparently uninformative messages affect asset market variables.

In our experiments, the effect of public messages actually depends on their reliability and content. In particular, we show that messages may affect asset price and bubble measures as long as they are not randomly generated. It seems that a minimum level of reliability is a necessary condition for messages to be effective. ${ }^{3}$ The reliability of a message can depend upon subject perceptions about how the message was generated. For example, subjects may perceive a preset message as being more reliable, ${ }^{4}$ than a randomly selected one, if they think that the content of the message depends on experimenter's past experience in dealing with similar situations.

The content of a message is the other decisive characteristic of an announcement. In our experiments we consider the following two messages: "The price is too high" or "The price is too low". We find that these messages do not have the same degree of effectiveness for a given level of reliability. For inexperienced subjects, the release of the preset message "The price is too high" reduces the amplitude and duration of bubbles whereas, the release of the message "The price is too low" is ineffective. The message "The price is too low", on the other hand, cancels the effect of experience on bubble dissipation for experienced subjects. We also show that the preset message "The price is too high" significantly increases the frequency with which asset prices rise.

Another necessary condition for messages to be effective is their consistency with subjects' beliefs. It is well known that asset market prices exhibit a positive trend from the start of the experiment. This may lead subjects to believe that asset prices are too high. In this case the message "The price is too high" confirms subjects' beliefs and reinforces their convictions. Contrarily, the message "The price is too low" is disregarded. This asymmetry in subject response to two different, and apparently uninformative, messages can be explained by the fact that they are effective when they are consistent with the prior beliefs of subjects. Indeed, when asset markets are repeated, almost half the subjects tend to believe that prices are too low, or correct. In line with this argument the strength of the message "The price is too high" decreases whereas, the saliency of the message "The price is too low" increases.

Further, we observe that the message, "The price is too high" has no effect upon bubbles. In addition, the effect of public messages on bubbles and asset prices is accompanied by a reduction in the magnitude of inconsistent bid/ask behavior. Interestingly, the message "The price is too low" eliminates the usually observed effect of experience on bubbles reduction for experienced subjects.

\section{Related literature}

Evidence of bubbles in experimental asset markets dates back to SSW. Prices in these markets tend to start below fundamental value (as determined by the expected dividend value of the asset) and quickly rise above the intrinsic value until a crash occurs in the final periods. Bubbles have been found to be robust to different treatments such as short selling, capac-

\footnotetext{
2 As a result, it can be the case that a fully reliable message is not informative.

3 We have to stress that this condition is weak since subjects may perceive preset announcements as reliable. Further, market-based messages are less effective than preset messages in reducing the magnitude of bubbles. This suggests that increasing the reliability of a message does not decrease bubbles further.

${ }^{4}$ Subjects may assume that these messages contain more information than is implied. In this sense we argue that preset messages are more reliable than randomly selected messages.
} 
Table 1

Levels of reliability of the message for different treatments.

\begin{tabular}{lll}
\hline Treatments & Source of the message & Reliability of the message \\
\hline $\mathbf{R H}, \mathbf{R L}$ & Random & Low \\
$\mathbf{H}, \mathbf{L}$ & Preset (by the experimenter) & Medium \\
$\mathbf{A}$ & Contingent (on asset market prices) & High \\
\hline
\end{tabular}

ity to buy on margin, brokerage fee, and a limit price change rule (King et al., 1993). SSW stress that the lack of common expectations could be an explanation for bubble formation. Porter and Smith (1995) introduce a futures market in experimental asset markets as a mechanism to promote common expectations among traders. They find that the introduction of a futures market significantly reduces bubble amplitude, although bubble duration is not significantly affected. A complete set of futures (one for each of the 15 periods) seems to eliminate bubbles (Noussair and Tucker, 2006). Bubbles can thus be explained by the uncertainty about others' behavior. Under the speculative hypothesis agents buy at prices higher than fundamentals in order to resell the asset to (possibly) irrational traders at a higher price. However, the probability of making speculative profits diminishes towards the end of the experimental market and prices revert to fundamentals.

A critical variable in the development of experimental bubbles is subject experience. The fact that the dividend probabilistic structure is common information is not sufficient to ensure common expectations among participants. Subjects tend to develop common expectations with experience. The result is that agents with long common experience trade at fundamental value. For example, bubbles tend to disappear with twice-experienced subjects even when experienced and inexperienced traders are mixed (Dufwenberg et al., 2005). However, bubbles may still be observed among twice-experienced traders when the market environment is modified by increasing liquidity and dividend uncertainty (Hussam et al., 2008).

Another possible explanation for bubble formation is subject irrationality (Lei et al., 2001). They consider an experimental design in which speculation is impossible. Subjects are either buyers or sellers but, cannot purchase to resell. The underlying hypothesis is that the lack of reselling should impede speculative behavior. They find that even in the absence of reselling, bubbles and crashes occur. Discarding the speculative hypothesis, the authors emphasize the importance of individual irrationality in explaining asset price patterns. They find evidence of systematic errors in decision making accompanying bubbles. Traders engage in unprofitable transactions at prices above the maximum possible or below the minimum possible dividend stream.

In this paper, we analyze how the release of messages in experimental asset markets can affect the patterns of asset prices and trading volumes. There are two mechanisms by which messages can impact market variables. First, messages may help irrational traders to discover how to compute the fundamental value of the asset. Second, messages may serve as focal points to coordinate traders' expectations. Rational traders may anticipate that messages can induce potentially irrational subjects to trade closer to fundamental values. As a result, the magnitude of speculation decreases and thus, bubbles are reduced.

\section{Experimental design}

Subjects were recruited from the undergraduate population at George Mason University. The experiments lasted $2-1 / 2 \mathrm{~h}$ and subjects earned an average of $\$ 34$, including a $\$ 7$ show-up fee. All experiments were computerized using common interactive instructions. ${ }^{5}$

We use a standard experimental asset market in which nine subjects trade a security with a finite life of 15 periods. At the end of each period, the asset pays a random dividend (in cents) drawn from a uniform distribution over $\{0,8,28,60\}$ giving an expected dividend of 24 cents per period. Of the nine traders, five were endowed with two shares and 550 cents in cash, while the other four were given an initial portfolio of four shares and 180 cents in cash. Note that, using backward induction, risk neutral agents should trade at the fundamental value of 360 cents in period one with decrements of 24 cents in each period thereafter (Tirole, 1982).

An experimental session involves two consecutive markets of 15 periods with the same nine subjects. In the first market, each trading period lasts for $180 \mathrm{~s}$ while the duration is $160 \mathrm{~s}$ in the subsequent market. ${ }^{6}$ All other characteristics of the environment are the same for the two markets. Subjects receive an electronic announcement in period 10 (in all markets) stating that there are only five periods remaining.

Subjects receive messages in periods 3,7 and 12 . These periods were chosen, because, from past asset market experiments we know that bubbles usually form between periods 3 and 7 while, crashes occur towards the last few periods. A message in period 12 would thus tell us if it has any impact on the crash whereas, messages in periods 3 and 7 should inform us about their effect upon bubble formation.

As mentioned before, messages are classified according to their content, reliability and timing. We consider the three messages illustrated in Table 1 (in increasing order of reliability). A contingent message is considered to be more reliable than a random and a preset message. A preset message, meanwhile, is considered to be more reliable than a random message. We argue that subjects may perceive that a preset message is based on experimenter experience with the environment and

\footnotetext{
${ }^{5}$ Instructions for the experiments can be found at http://esi2.chapman.edu/words/page1.html.

6 The second asset market was announced at the end of the first session.
} 
consider it to be more reliable. We consider treatments in which a message with qualitative content is released at arbitrary points in time. Maintaining the qualitative content and arbitrary timing of the announcement, we then vary the degree of reliability of the message. The table below summarizes the different treatments.

\subsection{Random message}

It is seems reasonable that markets would react to a message if the source were reliable, it was released at "the right time" and contained precise content. Contrarily, no reaction would be observed if a message lacked content, arrived at the incorrect moment and/or came from an unreliable source. Given this, a natural place to start would be the release of a message that we expect, a priori, to have no impact on market variables. In our opinion a random message fulfills these conditions.

Subjects were informed in the instructions about the procedure used to select the random message. They were told that depending upon the draw, they will receive one of the following two messages: "The price is too high" (henceforth RH for Random High message) or the "price is too low" (henceforth $\mathbf{R L}$ for Random Low message). ${ }^{7}$ The content of the message was selected by rolling a die in front of them at the beginning of the session. ${ }^{8}$ They were told that this message will be released in periods 3,7 and 12 . This is a weak treatment since messages are unreliable, the content of the message is qualitative and the timing of the message is arbitrary and known to market participants. The subjects receive the following instructions:

"Before the session begins, the experiment monitor will roll a die at the front of the room. If the number obtained by rolling the die is between 1 and 3 then the message "The price is too low" will appear on the screen in rounds 3, 7, and 12. If the number obtained by rolling the die is between 4 and 6 then the message "The price is too high" will appear on the screen in rounds 3,7 , and 12."

The message appears on the right hand corner of the trading screen as follows:

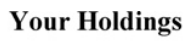

\begin{tabular}{|l|l|}
\hline Cash & 150 \\
\hline Shares & 3 \\
\hline
\end{tabular}

\section{THE PRICE IS TOO HIGH}

The dividend has equal chance of being $0,8,28$, or 60 cents this period.

This message is then repeated in the instructions summary. At the end of the instructions the subjects are further asked about the message. They answer the following question:

\section{Question 6}

In which period(s) will a randomly selected message saying that "The price is too high" or "The price is too low" be released:

$\circ$ A. Periods 3, 6 and 12

- B. Periods 3, 7 and 12

- C. Period 3

$\circ$ D. Never

This exercise stresses upon the subjects that the message is selected randomly and that it appears in known preselected periods. We thus ensure that the content and timing of the message are common information. ${ }^{9}$

\subsection{Preset message}

Subjects receive the following preset messages in the two treatments.

In treatment $\mathbf{L}$ subjects received the message:

"THE PRICE IS TOO LOW."

In treatment $\mathbf{H}$ subjects received the message:

"THE PRICE IS TOO HIGH."

\footnotetext{
${ }^{7}$ Instructions for this treatment can be found at http://esi2.chapman.edu/words/random/page1.html.

${ }^{8}$ In order to obtain exactly the same number of sessions with the message "The price is too high" and with the message "The price is too low" we were forced to release the message "The price is too low" in the last session independently of the result of the die.

${ }^{9}$ The content and the timing of the message are not common knowledge in the sense of SSW and Smith (2003) since subjects are not trained to use this piece of information. Subjects have common information ("knowledge that") about the content and the timing of the message but do not have common knowledge ("knowledge how") until they have experienced the effect of the message.
} 
Table 2

Experimental design.

\begin{tabular}{lll}
\hline Treatment & Message (periods 3, 7, and 12) & Number of sessions \\
\hline Baseline $(\mathbf{N})$ & No message & 3 inexperienced 3 experienced \\
Random Message $(\mathbf{R H})$ & "The price is too high" & 3 inexperienced 3 experienced \\
Random Message $(\mathbf{R L})$ & "The price is too low" & 3 inexperienced 3 experienced \\
Preset message $(\mathbf{H})$ & "The price is too high" & 3 inexperienced 3 experienced \\
Preset message $(\mathbf{L})$ & "The price is too low" & 3 inexperienced 3 experienced \\
Market-based Message $(\mathbf{A})$ & "The price is too low" when $P_{t}<f_{t}$ "The price is too high" when $P_{t}>f_{t}$ & 3 inexperienced 3 experienced \\
\hline
\end{tabular}

Table 3

Summary of the market environment.

\begin{tabular}{lll}
\hline Trading mechanism & Asset & Traders \\
\hline Computerized continuous double auction & 15-period asset Uncertain dividends & 9 traders \\
\hline
\end{tabular}

We inform the subjects about the content and the timing of a preset message in the instructions. ${ }^{10}$ The subjects receive the following instructions:

"Before the session starts, a preset message will be selected that says that either "The price is too high" or "The price is too low". The message will appear during the experiment, in the panel under Your Holdings, and it will only appear in periods 3, 7, and 12."

\subsection{Market-based message}

In the final treatment (treatment $\mathbf{A}$ ) the content of the message is contingent upon the situation of asset prices in the previous period. ${ }^{11}$ This increases its reliability without modifying the timing and the content. Subjects receive the message in periods 3, 7 and 12. They receive either one of the two messages, "The price is too high", or "The price is too low", depending upon whether the average price of the asset is strictly greater, or lower, than its fundamental value in the prior period.

The subjects are informed in the instructions about the exact process that determines the message. ${ }^{12}$ Table 2 outlines the experimental design with the different treatments. Where, $P_{t}$ and $f_{t}$, denote the average price of the asset and its fundamental value in period $t$, respectively.

All treatments are based on a common market environment described below (Table 3).

\section{Hypotheses}

Messages should never influence asset prices under the full rationality hypothesis. As a result, we expect the following hypothesis to hold for the experimental sessions with inexperienced subjects.

Hypothesis 1. (Bubble measures): Public messages do not affect bubble measures regardless of their reliability.

Hypothesis 2. (Price changes): Public messages do not affect asset prices in the periods in which they are released regardless of their reliability.

We also expect messages not to affect asset prices and bubbles measures for experienced subjects. At the same time, we should observe, as in previous experimental asset markets, that experience significantly reduces bubbles regardless of the release of public messages.

Hypothesis 3. (Experience):

(i) For experienced subjects, public messages neither affect bubble measures nor the direction of price changes.

(ii) Bubble measures are reduced with experienced subjects regardless of the release of public messages.

For messages to have any effect, one would have to assume that traders behave irrationally, or lack common knowledge of rationality. When traders exhibit irrational behavior, messages can affect trading patterns by facilitating the computation of the rational expectations equilibrium (Lei et al., 2001). Non-rationality may also manifest itself through the inability of agents to use backward induction (Katok et al., 2002; Johnson et al., 2002) in determining the equilibrium price (Tirole, 1982). Under these circumstances, messages that refer to the fundamental value of the asset may help subjects in determining its intrinsic value.

\footnotetext{
10 Similarly to the case of random messages, subjects are asked about the timing of the message in the quiz. Instructions for this treatment can be found at http://esi2.chapman.edu/words/page1.html.

${ }^{11}$ Marked-based messages are by definition always true implying a reliability level of $100 \%$.

12 Instructions for this treatment are available at http://esi2.chapman.edu/words/market/page1.html.
} 
Messages may matter when subjects are uncertain about the behavior of others. If this is the case then even traders capable of calculating the fundamental value of an asset may speculate, resulting in trades at prices higher than fundamentals (SSW). On the other hand, messages may reduce speculative activity if rational subjects anticipate that they help irrational subjects focus on the fundamental value of the asset. The release of messages can thus facilitate coordination of beliefs among traders with different levels of sophistication.

The experimental results are presented in section (V). We test Hypothesis 1 and Hypothesis 2 in this section and study the effect of messages on the different bubble measures (Section 5.1) and on the direction of asset prices changes (Section 5.2) for inexperienced subjects. This is then followed by the analysis of the effect of public messages in the case of once-experienced subjects (Section 5.3). This section provides a test for Hypothesis 3. We analyze inconsistent behavior in Section 5.4. Section 6 concludes.

\section{Experimental results}

Denote by $i j k$ the experimental session $j$ under treatment $i \in\{\mathbf{A}, \mathbf{H}, \mathbf{L}, \mathbf{N}, \mathbf{R H}, \mathbf{R L}\}$ with inexperienced $(k=1)$, or once-experienced $(k=2)$, subjects. Previous studies consider Amplitude, Duration, Normalized Average Price Deviation and Normalized Absolute Price Deviation as relevant measures of bubbles (Dufwenberg et al., 2005). ${ }^{13}$ We further add Upward Trend as a complementary measure of the impact of messages on market variables:

1. Amplitude: Measures the trough-to-peak change in asset value relative to its fundamental value. This is measured as, $A=\operatorname{Max}\left\{P_{t}-f_{t} / E(: t=1 \ldots 15\}-\operatorname{Min}\left\{P_{t}-f_{t} / E: t=1 \ldots 15\right\}\right.$. Where, $P_{t}$ is the average market price in period $t, f_{t}$ is the fundamental value of the asset in period $t$, and $E$ is the expected dividend value over the life of the asset.

2. Duration: Measures the length, in periods, in which there is an observed increase in market prices relative to the fundamental value of the asset. Formally, duration is defined as: $D=\operatorname{Max}\left\{m: P_{t}-f_{t}<P_{t+1}-f_{t+1}<\ldots<P_{t-m}-f_{t-m}\right\}$.

3. Normalized average price deviation (NAV): Sums up the absolute deviation between the average price and the fundamental value for each of the 15 periods. It is defined as follows: $N A V=\left(\Sigma\left|P_{t}-f_{t}\right| / 15\right) \mid P$.

4. Normalized absolute price deviation (NAP): As defined in Haruvy and Noussair (2006), NAP measures the per-share aggregate overvaluation (or undervaluation), relative to the fundamental value of the asset in a given period and is defined as: $N A P=\left(\Sigma\left|P_{i t}-f_{t}\right|\right) /(100 \times 26)$ where, $P_{i t}$ is the price of the $i$ th transaction in period $t, 26$ the total number of shares and 100 is a normalization scalar. Large values of NAP reflect volumetric deviations from fundamentals. This measure is similar to the normalized average price deviation. However, NAV does not depend on the number of trades and can then be used to compare the extent of mispricing in sessions with different levels of trading volumes.

5. Upward Trend: Measures the number of consecutive periods in which average prices $\left(P_{t}\right)$ increases. By definition, the Upward Trend is inferior to the Duration of the bubble since the bubble can grow when stock prices go down (given that the fundamental value of the asset decreases over time). Also, two experimental markets may have the same duration and still exhibit very different trends. Given that the messages released in our experiments refer to the level of asset prices we are interested in analyzing not only their evolution of mispricing but also their patterns.

We run the following seemingly unrelated regression (SUR) in order to assess the effects of different treatments for inexperienced subjects.

$$
\begin{aligned}
& \operatorname{AMPLITUDE}(i)=C+\beta_{\mathrm{H} 1, A M} \times \mathrm{H} 1(i)+\beta_{L 1, A M} \times \mathrm{L} 1(i)+\beta_{R H 1, A M} \times \mathrm{RH} 1(i)+\beta_{R L 1, A M} \times \mathrm{RL} 1(i)+\beta_{A, A M} \times \mathrm{A} 1(i) ; \\
& \operatorname{DURATION}(i)=C+\beta_{H 1, D U} \times \mathrm{H} 1(i)+\beta_{L 1, D U} \times \mathrm{L} 1(i)+\beta_{R H 1, D U} \times \mathrm{RH} 1(i)+{ }_{\beta R L 1, D U} \times \mathrm{RL} 1(i)+\beta_{A, D U} \times \mathrm{A} 1(i) \text {; } \\
& \mathrm{NAV}(i)=C+\beta_{H 1, N V} \times \mathrm{H} 1(i)+\beta_{L 1, \mathrm{NV}} \times \mathrm{L} 1(i)+\beta_{R H 1, N V} \times \mathrm{RH} 1(i)+\beta_{R L 1, N V} \times \mathrm{RL} 1(i)+\beta_{A, N V} \times \mathrm{A} 1(i) ; \\
& \mathrm{NAP}(i)=C+\beta_{H 1, N A} \times \mathrm{H} 1(i)+\beta_{L 1, N A} \times \mathrm{L} 1(i)+\beta_{R H 1, N A} \times \mathrm{RH} 1(i)+\beta_{R L 1, N A} \times \mathrm{RL} 1(i)+\beta_{A, N A} \times \mathrm{A} 1(i) ; \\
& \operatorname{TREND}(i)=C+\beta_{H 1, T R} \times \mathrm{H} 1(i)+\beta_{L 1, T R} \times \mathrm{L} 1(i)+\beta_{R H 1, T R} \times \mathrm{RH} 1(i)+\beta_{R L 1, T R} \times \mathrm{RL} 1(i)+\beta_{A, T R} \times \mathrm{A} 1(i) .
\end{aligned}
$$

The term $C$ stands for the constant and $i$ denote the session. The independent variables are dummy variables, taking a value of 1 if the session corresponds to the given treatment, and $\beta_{j, k}$ is a coefficient denoting treatment $j$ and bubble measure $k \in\{\mathbf{A M}, \mathbf{D U}, \mathbf{N V}, \mathbf{N A}, \mathbf{T R}\}$ (see Table 4 below).

\subsection{Messages and bubbles: inexperienced subjects}

In this section we test Hypothesis 1 under which public messages should not affect bubble measures.

Our first result constitutes a starting point for our analysis of the effect of public messages on asset market variables. We find that a random message does not significantly impact bubble measures. ${ }^{14}$ It seems that a minimum level of reliability is necessary for messages to affect asset prices. The results of SUR estimates are provided in Table 4.

\footnotetext{
13 We disregard Turnover as a measure of bubble. Our analysis of the effect of messages on trading volumes is available upon request.

14 The descriptive statistics of the various measures for the different treatments are provided in the appendix (Table A1).
} 
Table 4

SUR estimates for inexperienced traders.

\begin{tabular}{lccccc}
\hline Coefficients $(P$-values) & Amplitude $(\mathbf{A M})$ & Duration $(\mathbf{D U})$ & NAV $(\mathbf{N V})$ & NAP $($ NA) & Trend (TR) \\
\hline Intercept (N1) & $1.26\left(0.00^{* * *}\right)$ & $10.33\left(0.00^{* * *}\right)$ & $130.08\left(0.00^{* * *}\right)$ & $11.16\left(0.00^{* * *}\right)$ & $6.33\left(0.00^{* * *}\right)$ \\
Preset message (H1) & $-0.27\left(0.02^{* *}\right)$ & $-4.67\left(0.04^{* *}\right)$ & $-71.57\left(0.00^{* * *}\right)$ & $-6.85\left(0.00^{* * *}\right)$ & $-4.00\left(0.04^{* *}\right)$ \\
Preset message (L1) & $0.04(0.70)$ & $0.33(0.88)$ & $-1.33(0.92)$ & $-4.57\left(0.01^{* *}\right)$ & $-1.00(0.60)$ \\
Random message (RH1) & $-0.09(0.41)$ & $1.33(0.55)$ & $-14.28(0.30)$ & $-0.46(0.80)$ & $-1.33(0.48)$ \\
Random message (RL1) & $-0.12(0.28)$ & $1.00(0.66)$ & $-17.58(0.20)$ & $-1.60(0.38)$ & $-0.33(0.86)$ \\
Market-based message (A1) & $-0.19\left(0.09^{*}\right)$ & $-2.00(0.38)$ & $-24.69\left(0.076^{*}\right)$ & $-2.85(0.12)$ & $-1.67(0.38)$ \\
\hline
\end{tabular}

Table 5

Proportion of subjects choosing the message "The price is too high" in the three experimental sessions of treatment C.

\begin{tabular}{lcc}
\hline Period & Inexperienced subjects & Once-experienced subjects \\
\hline 3 & $67 \%$ & $67 \%$ \\
7 & $85 \%$ & $52 \%$ \\
12 & $67 \%$ & $41 \%$ \\
Average & $73 \%$ & $53 \%$ \\
\hline
\end{tabular}

\subsubsection{Result 1: Random message and bubble measures}

(i) The random message "The price is too high" does not significantly reduce Amplitude, Duration, Upward Trend, Normalized Average Price Deviation and Normalized Absolute Price Deviation.

(ii) The random message "The price is too low" does not significantly affect bubble measures.

One can see that the coefficients associated with RH1 and RL1 are not statistically significant at standard levels. This result shows that random messages have no effect upon the different bubble measures for inexperienced subjects. We now look at messages with higher levels of reliability. We first look at the case of the preset message. ${ }^{15}$

\subsubsection{Result 2: Preset message and bubble measures}

(i) The release of the message "The price is too high" significantly reduces the Amplitude, Duration, Normalized Average Price Deviation and Normalized Absolute Price Deviation for inexperienced subjects.

(ii) The release of the message "The price is too low" does not significantly affect the different measures of bubbles.

Result 2 refutes Hypothesis 1 by establishing that a preset message ("The price is too high") can affect bubble measures. The decrease in the magnitude of the bubbles (treatment $\mathbf{H}$ ) under the (preset) message "The price is too high" is statistically significant. However, we find no evidence that the preset message "The price is too low" exacerbates bubbles. ${ }^{16}$

Result 2 implies that, behavior is affected in an asymmetric manner when subjects are exposed to a preset message for the first time. The effect of preset messages on different bubble measures depends upon the message content (see SUR regression). In particular, we conjecture that messages are more effective when they are consistent with subjects' beliefs about the fundamental value of the asset. To verify this claim we elicit subject beliefs (Treatment $\mathbf{C}$ ) regarding the market price. Subjects had the possibility to select one of the following statements: "The price is too low", "The price is correct" or "The price is too high". Subjects report their beliefs about asset prices at the end of periods 3, 7 and 12 . We can see from Table 5 that the release of the message "The price is too high" is consistent with their beliefs. This could be due to the fact that the positive trend in the early periods may induce subjects to think that prices are too high. ${ }^{17}$ On the other hand, it could be the case that subjects have prior beliefs that prices are usually too high.

Results 1 and 2 are illustrated in Fig. 1. The Upward Trend is practically eliminated ( 2.3 periods on average for inexperienced traders) in treatment $\mathbf{H}$ compared to the baseline treatment in which the upward trend is equal to 6.3. In the next result we analyze the effect of market-based messages (treatment $\mathbf{A}$ ). The message subjects receive, in periods 3,7 and 12 , depends on the actual level of mispricing in the previous period.

\subsubsection{Result 3 (market-based message and bubble measures)}

The release of market-based messages significantly reduces the different bubble measures.

Result 3 is established by checking the significance of SUR estimates associated with A1 (Table 4). We find that the release of market-based messages significantly reduces the amplitude and NAV of asset markets bubbles. In Table 6, we provide the

\footnotetext{
15 Recall, that we argue that a preset message will be more reliable than a random one if subjects think that it incorporates experimenter's experience in dealing with similar markets.

16 This result is confirmed by the SUR estimates (Table 4) since the coefficients associated with H1 are all significant, whereas the coefficients associated with $\mathbf{L 1}$ are never significant except for the NAP coefficient.

17 When the increase in asset prices in the period preceding the release of a message is particularly high then the proportion of traders that express the belief The price is too highïs significantly higher in the period after the announcement. Our analysis of subject beliefs is available upon request.
} 


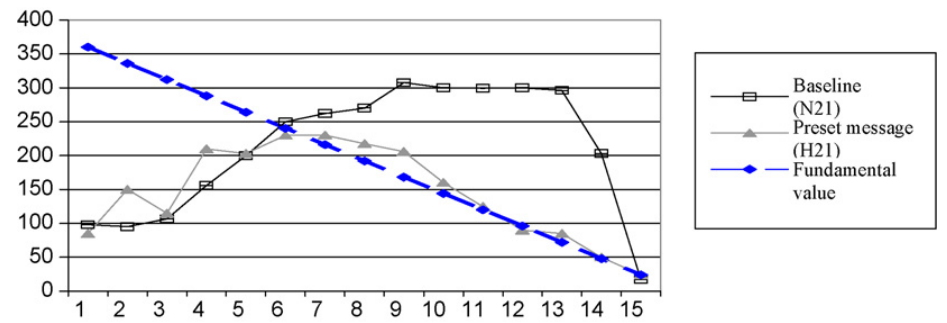

Fig. 1. Median prices per period for treatments N21 and H21.

Table 6

Average bubble measures for treatments $\mathbf{A}, \mathbf{H}$ and $\mathbf{N}$ Inexperienced subjects.

\begin{tabular}{llrrr}
\hline Average measures & Amplitude & Duration & NAV & NAP \\
\hline Baseline $(\mathbf{N})$ & 1.26 & 10.33 & 11.16 & 130.08 \\
Preset message $(\mathbf{H})$ & 0.98 & 5.67 & 4.31 & 58.51 \\
True message $(\mathbf{A})$ & 1.07 & 8.33 & 7.78 & 2.33 \\
\hline
\end{tabular}

Table 7

Messages released in treatment $\mathbf{A}$

\begin{tabular}{llllll}
\hline \multirow{2}{*}{ Period } & Session A11 & Session A21 & Session A31 & Session A12 & Session A22 \\
\cline { 2 - 5 } & Inexperienced & & & Experienced & $L$ \\
3 & $L$ & $L$ & $L$ & $L$ & $L$ \\
12 & $L$ & $L$ & $H$ & $H$ & $H$ \\
\hline
\end{tabular}

average bubble measures for treatments $\mathbf{A}, \mathbf{H}$ and $\mathbf{N}$. Compared to treatment $\mathbf{N}$, a significant reduction in the magnitude of bubbles is observed for treatment $\mathbf{A}$. We test this conjecture by comparing the different bubbles measures in treatments $\mathbf{A}$ and $\mathbf{H}$ with bubbles measures in the other treatments ( $\mathbf{L}, \mathbf{R H}, \mathbf{L}$ and $\mathbf{N}$ ). For statistical purposes, we decide to pool the data of treatments that do ( $\mathbf{A}$ and $\mathbf{H})$ or, do not $(\mathbf{R H}, \mathbf{R L}, \mathbf{L}$ and $\mathbf{N})$ affect, bubbles measures according to the SUR estimates. Having pooled the respective data, we then test whether, compared to treatments $\mathbf{R H}, \mathbf{R L}, \mathbf{L}$ and $\mathbf{N}$, the magnitude of bubbles is significantly lower in treatments $\mathbf{A}$ and $\mathbf{H}$. Our results show that the release of either market-based, or preset, message ("The price is too high") tends to reduce bubble magnitudes (Appendix-Table A2). Interestingly, increasing the reliability of a message does not seem to strengthen the impact of public messages on the different variables.

We stress the relevance of the content of a message by comparing the effect of messages with the same content but, with different levels of reliability. To achieve this we make use of the sequence of messages that is actually released to traders in treatment $\mathbf{A}$ and is described in the Table 7 ( $H$ denotes "The price is too high" and $L$ denotes "The price is too low").

Note that, for the inexperienced sessions (A12, A21 and A31) the message "The price is too low" is displayed for all treatments in period 3. The message "The price is too high" is, meanwhile, displayed for all the three treatments in period 12. Given the sequence of messages delivered in treatment A, we analyze Phase I (periods 1-11) and Phase II (periods 12-15), separately, for these three sessions. We then compute a measure of mispricing for the two phases taken separately, and for treatments A, $\mathbf{H}$ and $\mathbf{L}$. Given that the Normalized Average Price Deviation is not affected by possible differences in trading volumes, we use it as a measure of the magnitude of bubbles. In Table 8 we display the level of mispricing for the two market phases and for the three message treatments.

One can see that the level of mispricing in treatment $\mathbf{A}$ (Table 8) is similar to the level of mispricing in treatment $\mathbf{L}$ in the first phase of the market. However, in the second phase, the level of mispricing is similar to that in treatment $\mathbf{H}$. This suggests that the effect of treatment $\mathbf{A}$ on the magnitude of the bubbles is closely related to the content of the message.

This finding is consistent with the fact that, for inexperienced subjects, the bubble measures presented in Table 6 tend to be higher for treatment $\mathbf{A}$ than for $\mathbf{H}$. Indeed, the effective message "The price is too high" is only displayed in period 12 in treatment $\mathbf{A}$ (for inexperienced subjects), whereas, this message is released in periods 3,7 and 12 in treatment $\mathbf{H}$. The content of the message appears to be a crucial dimension in understanding the effectiveness of a message. This is true since traders tend to react in a similar fashion to messages that are predetermined and market-based as long as they have the same

Table 8

Average mispricing measures: Phase I and II inexperienced subjects.

\begin{tabular}{llll}
\hline & Market-based message $(\mathbf{A})$ & Preset message $(\mathbf{H})$ & Preset message $(\mathbf{L})$ \\
\hline Normalized average price deviation $(t>12)$ & 123.1 & 92.2 & 126.3 \\
Normalized Average Price Deviation $(t>12)$ & 69.8 & 56.7 & 133.9 \\
\hline
\end{tabular}


Table 9

Proportion of periods 3, 7 and 12 for which median prices increase ${ }^{\mathrm{a}}$.

\begin{tabular}{|c|c|c|c|c|c|c|}
\hline & $\begin{array}{l}\text { Baseline } \\
(\mathbf{N})\end{array}$ & $\begin{array}{l}\text { Preset message } \\
(\mathbf{H})\end{array}$ & $\begin{array}{l}\text { Preset message } \\
\text { (L) }\end{array}$ & $\begin{array}{l}\text { Random message } \\
(\mathbf{R H})\end{array}$ & $\begin{array}{l}\text { Random message } \\
(\mathbf{R L})\end{array}$ & $\begin{array}{l}\text { Market-based } \\
\text { message }(\mathbf{A})\end{array}$ \\
\hline Inexperienced subjects & $7 / 9$ & $2 / 8$ & $6 / 9$ & $6 / 6$ & $5 / 6$ & $8 / 9$ \\
\hline Experienced subjects & $4 / 8$ & $5 / 8$ & $7 / 9$ & $4 / 8$ & $7 / 8$ & $6 / 9$ \\
\hline
\end{tabular}

a We drop the data associated with the periods for which the median price is unchanged.

content. This stresses that messages from unreliable sources may affect asset market prices in a similar magnitude as reliable statements. In the next section we complement our results on bubble measures by studying the impact of announcements on asset prices in the periods in which they are released. ${ }^{18}$

\subsection{Public messages and price changes}

In this section, we test whether public messages do not affect the direction of price changes (Hypothesis 2) for inexperienced subjects.

\subsubsection{Result 4: Price changes}

(i) For inexperienced subjects, a decrease in median prices is significantly more frequent under treatment $\boldsymbol{H}$ than under the baseline treatment, for $t \in\{3,7,12\}$.

(ii) For inexperienced subjects, an increase in median prices is not (significantly) more frequent under treatment $L$, or RL, than under the baseline treatment, for $t \in\{3,7,12\}$.

The preset message "The price is too high" significantly affects the direction of price changes. Our analysis thus leads us to reject Hypothesis 2. Indeed, as long as the message is not random (Appendix-Table A3), the number of periods for which asset prices decrease is significantly reduced by the release of the message, "The price is too high". Finally, the message "The price is too low" does not affect the direction of price changes whether it is preset or random. ${ }^{19}$ We present Result 4 in Table 9.

\subsection{Messages and bubbles: experienced subjects}

In this section we test Hypothesis 3 by analyzing how messages can affect market variables when subjects are experienced in the message. Our results are summarized below.

\subsubsection{Result 5 (messages and experience)}

(i) For experienced subjects, the preset message, "The price is too high", or market-based messages do not significantly reduce the different measures of bubbles.

(ii) For experienced subjects, increases in median prices are significantly more frequent under treatments $L$ and $R L$, than under treatments $N, H$ and $R H$, for $t \in\{3,7,12\}$. However, a decrease in median prices is not significantly more frequent under treatment $H$ than under the baseline treatment, for $t \in\{3,7,12\}$.

(iii) Whether preset, or randomly selected, the message "The price is too low" prevents experience from abating the different bubble measures.

\subsubsection{Support for Result 5 (i)}

Relative to the baseline treatment, the magnitude of bubbles is not reduced in treatments $\mathbf{A}$ and $\mathbf{H}$ with experienced subjects. One can see this by comparing the magnitude of the SUR coefficients associated with the variables N2, H2 and A2 in Table 10 .

With experienced subjects, the absence of the effect of the message in treatments $\mathbf{A}$ and $\mathbf{H}$ is confirmed by running a non-parametric Rank Sum test that compares the various bubble measures for the two treatments against the baseline-, and random, message treatments (RH \& RL). For once-experienced subjects (Appendix-Table A4), we confirm that the level of mispricing in treatments $\mathbf{A}$ and $\mathbf{H}$ is not significantly different from treatments $\mathbf{N}, \mathbf{R H}$ and $\mathbf{R L}{ }^{20}$ This result supports the first

\footnotetext{
18 Additional analyses concerning the impact of messages on bids and asks in the three periods in which messages are actually released are also available upon request.

19 Additional results on the effect of public messages on the direction of price changes are available upon request. In particular, we provide an analysis that includes market-based messages. In the current section we do not include market-based messages as they induce the release of both messages "The price is too high" and "The price is too low" making our analysis inappropriate.

20 Notice that we find that the difference in Normalized Average Price Deviation is marginally significant. However, this result is driven by session R21 (where NAV = 17.45) where the NAV is five to six times larger than in other sessions.
} 
Table 10

SUR estimates with once-experienced subjects.

\begin{tabular}{|c|c|c|c|c|c|}
\hline Coefficients ( $P$-values) & Amplitude (AM) & Duration (DU) & NAV (NV) & NAP (NA) & Trend (TR) \\
\hline $\begin{array}{l}\text { Intercept Inexperienced baseline, } \\
\text { preset low, random message (N1 and } \\
\mathbf{L 1} \text { and } \mathbf{R H 1} \text { and } \mathbf{R L 1})\end{array}$ & $1.22\left(0.00^{* * *}\right)$ & $11.00\left(0.00^{* * *}\right)$ & $121.78\left(0.00^{* * *}\right)$ & $9.51\left(0.00^{* * *}\right)$ & $5.67\left(0.00^{* * *}\right)$ \\
\hline $\begin{array}{l}\text { Inexperienced preset message (H1) } \\
\text { and market message (A1) }\end{array}$ & $-0.19\left(0.08^{*}\right)$ & $-4.00\left(0.02^{* *}\right)$ & $-39.83\left(0.00^{* * *}\right)$ & $-3.19\left(0.03^{* *}\right)$ & $-2.17\left(0.04^{* *}\right)$ \\
\hline Experienced baseline (N2) & $-0.29\left(0.04^{* *}\right)$ & $-3.00(0.15)$ & $-38.40\left(0.02^{* *}\right)$ & $-6.15\left(0.00^{* * *}\right)$ & $-2.00(0.15)$ \\
\hline Experienced preset message $(\mathbf{H} 2)$ & $-0.40\left(0.00^{* * *}\right)$ & $-2.67(0.21)$ & $-39.10\left(0.02^{* *}\right)$ & $-7.39\left(0.00^{* * *}\right)$ & $-3.33\left(0.02^{* *}\right)$ \\
\hline Experienced random message (RH2) & $-0.41\left(0.00^{* * *}\right)$ & $0.00(0.99)$ & $-38.04\left(0.02^{* *}\right)$ & $-1.32(0.48)$ & $-1.67(0.23)$ \\
\hline Experienced preset message (L2) & $-0.01(0.94)$ & $-2.67(0.21)$ & $-0.82(0.96)$ & $-6.40\left(0.00^{* * *}\right)$ & $-1.33(0.34)$ \\
\hline Experienced random message (RL2) & $0.04(0.77)$ & $-1.33(0.53)$ & $22.83(0.15)$ & $-4.98\left(0.01^{* * *}\right)$ & $-0.33(0.81)$ \\
\hline Experienced market message (A2) & $-0.35\left(0.01^{* *}\right)$ & $-2.33(0.27)$ & $-36.04\left(0.03^{* *}\right)$ & $-6.40\left(0.00^{* * *}\right)$ & $-2.33\left(0.09^{*}\right)$ \\
\hline
\end{tabular}
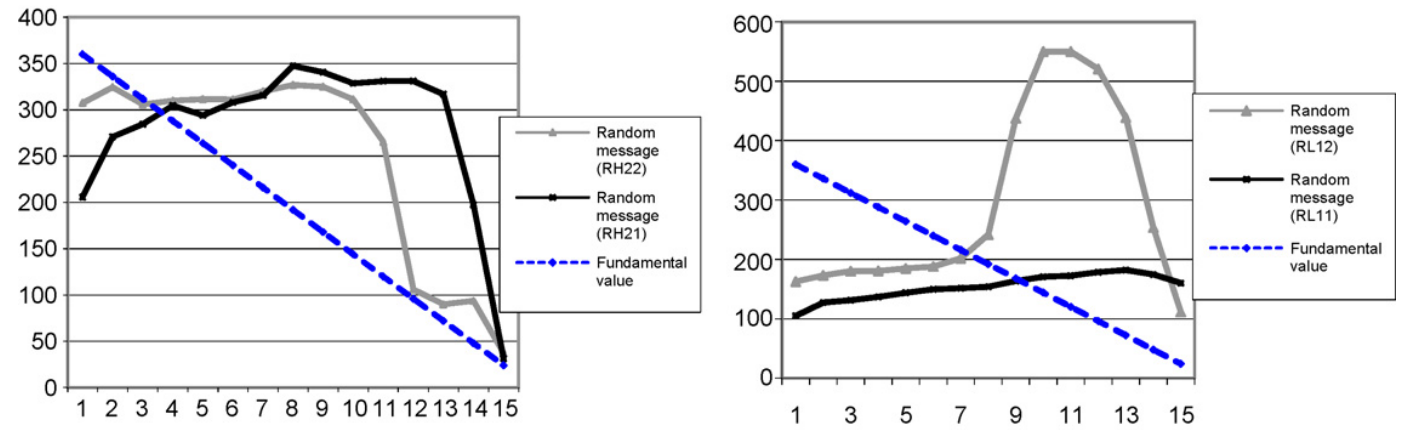

Fig. 2. Median prices per period for treatments RH21 and RH22 and for treatments RL11 and RL12.

part of Hypothesis $3(i)$ as subject experience eliminates, rather than reinforce, the effect of the public message on bubble measures.

\subsubsection{Support for Result 5 (ii)}

Whether preset, or random, the message "The price is too high" does not significantly affect the direction of price changes in the case of inexperienced subjects (Appendix-Table A3). However, the release of the message "The price is too low" tends to increase significantly the frequency with which median prices rise (Appendix-Table A3). This result refutes the second part of Hypothesis 3(i).

\subsubsection{Support for Result 5 (iii)}

Result 5 (iii) follows from the SUR estimates (Table 10). Except for Normalized Average Price Deviation, the coefficients associated with $\mathbf{L} 2$ and $\mathbf{R L 2}$ are not statistically significant at standard levels. This tells us that subjects experienced in the message ("The price is too low") fail to disregard the announcement. It is in this context that bubbles are not significantly reduced by experience. This result is interesting given the extensive amount of experimental evidence stating that subject experience systematically abates bubbles. Ours is the first paper that shows this without altering any other experimental parameters. ${ }^{21}$ Further, note that, in our experiments subjects react to messages even when they are experienced with the same randomly selected message (treatment $\mathbf{R L}$ ).

This result is confirmed by running a non-parametric Rank Sum test. We cannot reject the null hypothesis that, compared to treatments L1 and RL1, a given measure of mispricing is the same under treatments L2 and RL2 (Appendix-Table 5). Except for NAP, in treatments $\mathbf{L}$ and $\mathbf{R L}$, subject experience does not reduce bubble measures. NAP is significantly reduced with experience mostly because trading volumes drop drastically with once-experienced subjects.

The absence of any effects due to subject experience in treatment $\mathbf{L}$ and $\mathbf{R} \mathbf{L}$ is illustrated in Fig. 2 where we display the median prices for treatments $\mathbf{R H 2}$, and RL1, respectively. The Fig. on the left shows us the commonly observed effect of experience on the magnitude of bubbles. The figure on the right, meanwhile, shows that bubbles can survive even with experienced subjects when the message "The price is too low" is released.

In order to account for the effect of the message "The price is too low", we refer to the change in beliefs that occur when subjects are experienced (see Table A5 in Section 5.1). A great majority of inexperienced subjects believe that asset prices are too high whereas this belief is mitigated when subjects become experienced. As a result, we conjecture that the message

\footnotetext{
21 Hussam et al. (2008) show this by changing the dividend distribution and cash endowments. Also, Isaac and James (2000) show that changing the payment structure of subjects to a tournament can lead mispricing to persist with experience.
} 
"The price is too low" is more effective in experimental sessions with experienced subjects because, relative to inexperienced sessions, traders beliefs that "The price is too low" are stronger in experienced sessions. ${ }^{22}$ Contrarily, and as shown in Result 5 (i), the effect of the message "The price is too high" diminishes with experienced subjects. Summarizing, it seems that subjects do not change their beliefs due to the messages but, do use them to confirm their prior beliefs.

In the following section we study the effect of public messages on the occurrence of inconsistent patterns of trading. The objective is to analyze whether the effect of public messages can be explained by the effectiveness of the announcements in limiting subjects' irrational trading behavior. Indeed, for messages to have any effect, one would have to assume that traders behave irrationally, or lack common knowledge of rationality. When traders exhibit irrational behavior, messages can affect trading patterns by facilitating the computation of the rational expectations equilibrium (Lei et al., 2001). Also, messages may affect rational traders' behavior if the latter anticipate that public announcements may help irrational subjects focus on the fundamental value of the asset.

\subsection{Inconsistent behavior and speculative motive ${ }^{23}$}

Messages may help subjects with different levels of sophistication coordinate their beliefs. For example, messages may reduce speculative activity if rational subjects anticipate that they help irrational subjects compute the fundamental value of the asset. In this section we assess whether the impact of messages relies on subjects inconsistent patterns of trading, speculation motive or, both at the same time. First, we define subjects' trading behavior as being inconsistent in a given period whenever they are willing to sell the asset at a price lower than what they would be willing to pay for it. The analysis follows the definitions.

Definition 1. A subject exhibits inconsistent trading behavior in a given period if his minimum offer to sell is strictly lower than his maximum offer to buy.

Define, $I j$ as a measure of subject $j$ 's inconsistency and let, $I j$ be the sum of all periods for which subject $j$ exhibits inconsistent behavior. This would then imply that $I j$ equals 15 for fully inconsistent subjects. Contrarily, $I j$ is 0 when subjects are fully rational.

To analyze whether traders with different levels of inconsistency react differently to messages, we define a subject's overreaction to a message as follows.

Definition 2. A subjects "overreacts" to the message "The price is too high" ("The price is too low") when he reduces (increases) his minimum offer to sell (maximum offer to buy) by a magnitude that is greater than $24(-24)$ in the period in which the message is released.

Notice that 24 correspond to the expected dividend value of the asset in any given period. One needs to correct for the expected dividend value of the asset in order to compare offers to buy and sell in two consecutive periods. The difference in fundamental values of the asset between two consecutive periods is exactly equal to 24 . We denote $P R j$ as the variable that measures the number of times subject $j$ actually reacts to a message, where, $P R j \in\{0,1,2,3\}$.

\subsubsection{Result 6}

The release of the preset message "The price is too high", or the market-based message, significantly reduces the magnitude of inconsistent behavior for inexperienced subjects.

The average level of inconsistency $(I j)$ for the twenty-seven subjects in the baseline sessions is 3.7 and, 2.1 and 2.9, for treatments $\mathbf{H}$ and $\mathbf{A}$ (54 subjects), respectively. This difference is statistically significant (Appendix-Table A6).

Interestingly, we reject any difference in subjects' inconsistent behavior between treatments in the first two periods of all experimental sessions. This suggests that the decrease in the magnitude of consistent behavior can only be explained by the release of messages in periods 3,7 and 12 . Further, and compared to the baseline treatment in the same periods, inconsistent behavior is significantly reduced in treatments $\mathbf{H}$ and $\mathbf{A}$ (Appendix-Table A6). In the next figure we display the evolution of the proportion of inconsistent traders per period (Fig. 3).

We analyze the relationship between the degree of inconsistency of the traders and their reaction to messages. We first identify unsophisticated traders by the inconsistency of their trading pattern. We then assess the relationship between the level of inconsistency of traders and their reaction to messages. To control for possible endogeneity between subjects' inconsistent behavior and their reaction to messages, we first measure subjects' inconsistency in the first two periods (where no messages are actually released). This measure of inconsistency for a subject $j(I 2 j)$ takes values 0,1 or 2 . Note that, sophisticated traders are characterized by the absence of early inconsistent behavior, i.e. $I 2 j=0$, and unsophisticated traders are such that $I 2 j>0$. The proportion of sophisticated traders is equal to $33 \%$ and $41 \%$ for treatments $\mathbf{A}$ and $\mathbf{H}$, respectively. We can then show the following.

\footnotetext{
${ }^{22}$ It is also possible that the randomness of the message is less salient in the second session than in the first. Indeed, the draw of the die is implemented only once and just before the start of the first session.

${ }^{23}$ This analysis follows from the comments of one of the referees regarding irrational behavior and the effect of public messages.
} 


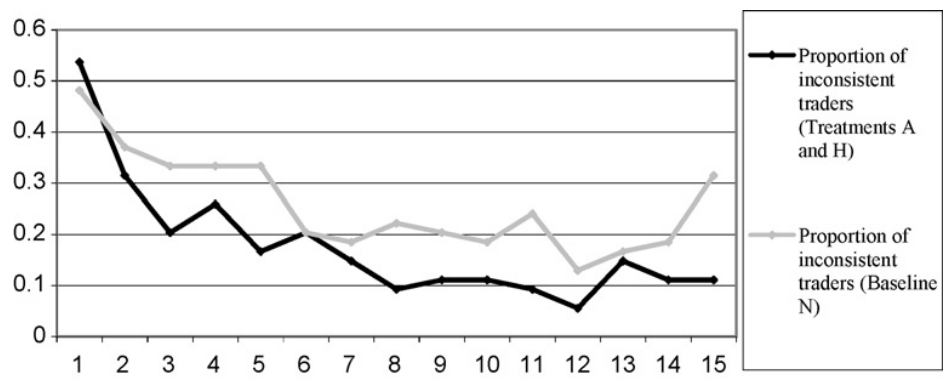

Fig. 3. Evolution of the proportion of inconsistent traders per period.

\subsubsection{Result 7}

For inexperienced subjects, the reaction of sophisticated traders' to the preset message "The price is too high", or market-based messages, is significantly stronger than for unsophisticated traders.

The average price reaction of sophisticated subjects in treatments $\mathbf{A}$ and $\mathbf{H}$ is twice as large $(P R j=1.2)$ than for unsophisticated subjects. Using a two-sided Wilcoxon Rank Sum test we see that the difference is significant $(Z=-2.4295$, $P$-value $=0.0151$ ). We can thus conclude that the reaction to messages cannot be uniquely explained by the presence of unsophisticated subjects that use the message to help them assess the fundamental value of the asset. Indeed, it seems that the strategy followed by sophisticated subjects is crucial in understanding the effect of messages. In particular, sophisticated traders may react to messages because they expect less sophisticated traders to be impacted by these announcements.

\subsubsection{Result 8}

For experienced subjects, the release of the preset ("The price is too high"), or market-based, message does not significantly reduce the magnitude of subjects' inconsistent behavior.

The average level of inconsistency $(I j)$ for the twenty-seven experienced subjects involved in the baseline sessions is 1.4 while, it is 1 and 1.5, in treatments $\mathbf{H}$ and $\mathbf{A}$ (54 subjects), respectively. This difference is not statistically significant (Appendix-Table A6). This suggests that the level of inconsistency with experienced subjects is particularly low.

In the first two periods only eleven out of the 54 subjects in message treatments $\mathbf{A}$ and $\mathbf{H}$ exhibit some degree of inconsistency in at least one of the two periods. This proportion of inconsistent traders, $20 \%$, contrasts with the sessions with inexperienced subjects where $63 \%$ of the traders exhibited some inconsistent behavior in the first two periods. The reduced level of inconsistency that characterizes sessions with experienced subjects tends to limit the effect of public messages. As a result, and similar to the conclusions of Lei et al. (2001), ${ }^{24}$ we conclude that the effect of public announcements is due both to the un-sophistication of traders and the fact that sophisticated traders anticipate the reaction of unsophisticated traders to public messages.

\section{Conclusion}

In this paper we explore the effects of qualitative announcements on asset market variables. Our first main result is that bubbles can be abated by releasing appropriate messages for inexperienced subjects. Releasing the preset message "The price is too high" at arbitrary points in time significantly reduces the magnitude of bubbles for inexperienced subjects. Contrarily, the message "The price is too low" does not affect the patterns of asset prices for inexperienced traders. Our results emphasizes that the content of a message is an important component that determines its effectiveness.

Our second main result is that public messages are especially effective when they confirm subjects' beliefs. In line with this we find that the message "The price is too low" prevents subjects' experience from reducing the magnitude of bubbles. Indeed, as asset prices decrease, this message tends to be more effective as it confirms subjects' beliefs that prices are actually too low.

We also investigate whether increasing the reliability of the message reduces bubbles further. This is tested by releasing announcements that depend on the actual level of mispricing of the asset. We find that the effect of market-based messages is comparable to the release of a preset message. This confirms our assertion that subjects view preset messages as reflecting experimenter experience in similar markets.

Finally, we study inconsistent patterns of trading in asset markets with public messages and show that messages may help subjects with different levels of sophistication to coordinate actions. Indeed, the magnitude of inconsistency is significantly decreased for inexperienced subjects when the message, "The price too high", is released.

Do our experiments have any implication for financial markets? Should there be a public policy answer to such announcements? These are difficult questions to which we cannot claim to have answers. Announcements are a part of the information

\footnotetext{
24 They argue that both irrationality and speculation explain the development of bubbles.
} 
fabric of financial markets. Our experiments show that experienced traders do a better job at filtering announcements. However, any announcement that agrees with trader beliefs is bound to generate greater reaction. This can occur regardless of trader experience. In this scenario trader experience matters less and may result in overreaction on their part. This may suggest the importance of institutional messages in steadying markets. ${ }^{25}$ For example, a joint reply by the European Community did a lot to steady the attack on the Euro recently. Clearly, policy makers and central bankers need to understand that clear messages from competent authorities have an important effect on markets. Indeed, if the authority is credible enough it may override the prior beliefs of the traders. Our analysis suggests that clear messages from reliable sources should be a policy position.

Our findings on the impact of messages on market variables open new directions of research concerning the relative importance of the different characteristics of public statements. For example, we could release messages with a precise content or we could improve the timing of the message. In that case, would it be possible to eliminate bubbles? Or, would it be possible to exacerbate bubbles? If so, would this effect be robust to traders' experience? We analyze some of these questions in future research.

\section{Appendix A. Supplementary data}

Supplementary data associated with this article can be found, in the online version, at doi:10.1016/j.jebo.2010.06.014.

\section{References}

Dufwenberg, M., Lindqvist, T., Moore, E., 2005. Bubbles and experience: an experiment. American Economic Review 95, $1731-1737$.

Forsythe, R., Palfrey, T., Plott, C., 1982. Asset valuation in an experimental market. Econometrica 50, 537-568.

Haruvy, E., Noussair, C., 2006. The effect of short selling on bubbles and crashes in experimental spot asset markets. Journal of Finance 61, $1119-1157$.

Hussam, R., Porter, D., Smith, V., 2008. Thar she blows: can bubbles be rekindled with experienced subjects? American Economic Review 98, 924-937.

Isaac, M., James, D., 2000. Asset market efficiency: the effects of tournament incentives for individuals. American Economic Review 90, 995-1004.

Johnson, E., Camerer, C., Sen, S., Rymon, T., 2002. Detecting failures of backward induction: monitoring information search in sequential bargaining. Journal of Economic Theory 104, 16-47.

Katok, E., Sefton, M., Yavas, A., 2002. Implementation by iterative dominance and backward induction: an experimental comparison. Journal of Economic Theory 104, 89-103.

King, R., Smith, V., Williams, A., Van Boening, M., 1993. The Robustness of Bubbles and Crashes in Experimental Stock Markets. Nonlinear Dynamics and Evolutionary Economics. Oxford University Press.

Lei, V., Noussair, C., Plott, C., 2001. Nonspeculative bubbles in experimental asset markets: lack of common knowledge of rationality vs. actual irrationality. Econometrica 69, 831-859.

Noussair, C., Tucker, S., 2006. Futures markets and bubble formation in experimental asset markets. Pacific Economic Review 11, 167-184.

Petersen, M., 2004. Information: Hard and Soft, Working Paper, Kellogg School of Business, Northwestern University.

Plott, C., Sunder, S., 1982. Efficiency of controller security markets with insider information: an application of rational expectation models. Journal of Political Economy 90, 663-698.

Plott, C., Sunder, S., 1988. Rational expectations and the aggregation of diverse information in laboratory security markets. Econometrica 56, $1085-1118$.

Porter, D., Smith, V., 1995. Futures contracting and dividend uncertainty in experimental asset markets. Journal of Business 68, 509-541.

Smith, V., 2003. Constructivist and ecological rationality in economics. American Economic Review 93, 465-508.

Smith, V., Suchanek, G., Williams, A., 1988. Bubbles, crashes and endogenous expectations in experimental spot asset markets. Econometrica 56, $1119-1151$.

Sunder, S., 1992. Market for information: experimental evidence. Econometrica 60, 667-695.

Tirole, J., 1982. On the possibility of speculation under rational expectations. Econometrica 50, 1163-1182.

25 A good example is the recent offensive launched by the Spanish government to restore its credit worthiness. 